\title{
Optimal tuning and calibration of bendable mirrors with slope measuring profilers
}

\author{
Wayne R. McKinney \\ Lawrence Berkeley National Laboratory, Berkeley, CA 94720, USA \\ Lawrence Berkeley National Laboratory, \\ 1 Cyclotron Road, M/S 2R0400, \\ Berkeley, CA 94720-8199, USA \\ $+1-510-495-4395$ TEL \\ $+1-510-486-7696$ FAX
}

Email address: WRMcKinney@lbl.gov

Jonathan 1. Kirschman

Lawrence Berkeley National Laboratory, Berkeley, CA 94720, USA

Lawrence Berkeley National Laboratory,

1 Cyclotron Road, M/S 2R0400,

Berkeley, CA 94720-8199, USA

$+1-510-495-4117$ TEL

+1-510-486-7696 FAX

Email address: jlkirsc@gmail.com

Alastair A. MacDowell

Lawrence Berkeley National Laboratory, Berkeley, CA 94720, USA 
Lawrence Berkeley National Laboratory,

1 Cyclotron Road, M/S 2R0400,

Berkeley, CA 94720-8199, USA

+1-510-495-4276 TEL

$+1-510-486-7696$ FAX

Email address: AAMacDowell@1bl.gov

Tony Warwick

Lawrence Berkeley National Laboratory, Berkeley, CA 94720, USA

Lawrence Berkeley National Laboratory,

1 Cyclotron Road, M/S 2R0400,

Berkeley, CA 94720-8199, USA

$+1-510-495-5919$ TEL

$+1-510-486-7696$ FAX

Email address: T_Warwick@lbl.gov

Valeriy V. Yashchuk

Lawrence Berkeley National Laboratory, Berkeley, CA 94720, USA

Lawrence Berkeley National Laboratory,

1 Cyclotron Road, M/S 2R0400,

Berkeley, CA 94720-8199, USA

+1-510-495-2592 TEL

+1-510-486-7696 FAX 
Email address: VVYashchuk@lbl.gov

*Corresponding author: wrmckinney@lbl.gov

Keywords: x-ray, mirror, synchrotron radiation, adaptive optics, elliptical bender, Long Trace

Profiler, LTP, surface slope measurement 


\begin{abstract}
We describe a technique to optimally tune and calibrate bendable x-ray optics for submicron focusing. The focusing is divided between two elliptically cylindrical reflecting elements, a Kirkpatrick-Baez (KB) pair. Each optic is shaped by applying unequal bending couples to each end of a flat mirror. The developed technique allows optimal tuning of these systems using surface slope data obtained with a slope measuring instrument, the long trace profiler (LTP). Due to the near linearity of the problem, the minimal set of data necessary for the tuning of each bender, consists of only three slope traces measured before and after a single adjustment of each bending couple. The data are analyzed with software realizing a method of regression analysis with experimentally found characteristic functions of the benders. The resulting approximation to the functional dependence of the desired shape provides nearly final settings. Moreover, the characteristic functions of the benders found in the course of tuning, can be used for retuning to a new desired shape without removal from the beamline and re-measuring. We perform a ray trace, using profiler data for the finally tuned optics, predicting the performance to be expected during use of the optics on the beamline.
\end{abstract}

\title{
1. Introduction
}

A primary goal of 3rd generation synchrotron light sources has been to achieve small spot sizes, preserving the brightness of the source all the way along the beam line to the sample. Zone plates, ${ }^{1}$ special x-ray lenses, ${ }^{2}$ and mirrors ${ }^{3}$ have been used successfully. At the Advanced Light Source (ALS) the focusing is divided in the tangential and sagittal directions into two elliptically cylindrical reflecting elements, the so-called Kirkpatrick-Baez $(\mathrm{KB})$ pair. ${ }^{4}$ Because fabrication of elliptical surfaces is complicated, the cost of directly fabricated tangential elliptical cylinders is often prohibitive. This is in contrast to flat optics, that are simpler to manufacture and easier to 
measure by conventional interferometry. The figure of a flat substrate can be changed by placing torques (couples) at each end. Equal couples form a tangential cylinder, and unequal couples can approximate a tangential ellipse or parabola.

In Sec. 2, we review the nature of the bending, and propose a new technique for optimal tuning of bendable mirrors before installation in the beamline. The technique adapts a method previously used to adjust mirrors on synchrotron radiation beamlines. ${ }^{5}$ However, in our case, optimal tuning of a bendable mirror is based on surface slope trace data obtained with a slope measuring instrument, in our case, the long trace profiler (LTP). We show (Sec. 2) that due to the near linearity of the bending problem, the minimal set of data necessary for tuning of two benders, consists of only three slope traces measured before and after a single adjustment of each bending couple. We provide an algorithm that was used in dedicated software for finding optimal settings for the mirror benders. The algorithm is based on a method of regression analysis with experimentally found characteristic functions of the benders. The resulting approximation to the functional dependence of the desired slope shape provides nearly final settings for the benders. Moreover, the characteristic functions of the benders found in the course of tuning, can be used for retuning of the optics to a new desired shape without removing from the beamline and remeasuring with the LTP. In Sec. 3 we provide a reduced form, but more intuitive implementation of our method. In this case, we subdivide the mirror into three regions, fit a circle to each subregion, and also fit a circle to the entire surface. The near linear dependences of the found curvatures on settings of the mirror benders allow rapid finding of the optimal settings via a simple linear extrapolation that can be done just graphically. Even the reduced method allows rapid iterative adjustment of both bending couples, and is typically much faster and more accurate than a random walk accomplished by fitting the surface heights to an evolving elliptical 
shape. The result of practical use of the developed technique to precisely tune a KB mirror used at the ALS for micro-focusing is presented In Sec. 4. In Sec. 4, we also describe a simple ray trace using the profiler data which shows expected performance in the beamline. This ray trace allows us to monitor the adjustment at each step of the process, if necessary; and provides confirmation of proper adjustment at the end of the procedure. In summary (Sec. 5) we discuss the next steps in the systematic improvement of optical performance for the application of $\mathrm{KB}$ pairs in synchrotron beamlines.

\section{Basis of the proposed technique to tune bendable mirrors}

Bendable mirrors have been well described in the literature. ${ }^{6,7}$ The curvature,

$$
\operatorname{Cur}(x) \equiv \frac{1}{R(x)} \equiv \frac{d^{2} y}{d x^{2}},
$$

as a function of position along a loaded beam is governed by the differential equation: ${ }^{8}$

$$
E I(x) \frac{d^{2} y}{d x^{2}}=M(x),
$$

where $x$ is the coordinate along the beam, the tangential direction; $y$ is in the direction of the deflection of the beam; $E$ is Young's modulus of the mirror material; $I(x)$ is the moment of inertia as a function of position along the beam, or mirror; $M(x)$ is the bending moment; and $R(x)$ is the radius of surface curvature.

As an example, we consider a mirror, bent in the tangential direction to form part of an elliptical cylinder, Fig. 1. It remains flat (neglecting anticlastic effects ${ }^{9}$ ) in the sagittal direction which is into/out of the paper. Such a mirror is used for the imaging of an object placed in the 
first (object) focus of the ellipse at the distance $r$ from the mirror center to the second (image) focus at distance $r^{\prime}$ from the mirror center. As drawn in Fig. 1, the downstream (right) end of the mirror is more curved than the upstream (left) end. This is reversed when $r^{\prime}>r$, but most beamline applications involve de-magnification. The parameters $r, r^{\prime}$ and $\theta$, the grazing angle of the incident ray at the center (pole) of the optic, uniquely specify the ellipse.

With two end bending couples, $C_{1}$ and $C_{2}$ (Fig. 2), a precise elliptical shape may be obtained by varying the moment of inertia of the substrate, $I(x)$, e.g., by varying its cross section. $^{7}$ In this case, the bending moment $M(x)$ in (1) will change linearly from $C_{1}$ at one mirror edge to the $C_{2}$ at another edge: ${ }^{6,7}$

$$
E I(x) \frac{d^{2} y}{d x^{2}}=\frac{C_{1}+C_{2}}{2}-\frac{C_{1}-C_{2}}{L} x,
$$

where $L$ is the length of the mirror. As we have mentioned above, equal couples make a cylindrical mirror. Other schemes can approximate a parabola, as an extremum of the elliptical mirror.

With straightforward transformations, the basic relation can be written:

$$
\frac{d^{2} y}{d x^{2}}=C_{1} g_{1}(x)+C_{2} g_{2}(x)
$$

where we have defined:

$$
g_{1}(x) \equiv\left(\frac{1}{2}-\frac{1}{L} x\right) \frac{1}{E I(x)} \text { and } g_{2}(x) \equiv\left(\frac{1}{2}+\frac{1}{L} x\right) \frac{1}{E I(x)} .
$$


Integrating (4) to get the slope from the curvature,

$$
\alpha(x, \hat{C}) \equiv \frac{d y}{d x}=C_{0}+C_{1} f_{1}(x)+C_{2} f_{2}(x),
$$

where

$$
f_{1}(x)=\int g_{1}(x) d x \text { and } f_{2}(x)=\int g_{2}(x) d x
$$

$C_{0}$ is the necessary constant of integration that is the overall tilt of the mirror.

According to Eq. (6), the slope of a bendable mirror is a linear combination of two functions, $f_{1}(x)$ and $f_{2}(x)$, characteristic of the particular mirror design. Due to the linearity, the error function in the mirror slope distribution, which appears at mis-tuned couples, with respect to the ideal (desired) surface is linear in these to be determined characteristic functions. The mirror shape optimization consists in finding the optimal values of parameters $C_{1}$ and $C_{2}$ that correspond to the minimum of the errors evaluated as a root mean square (rms) deviation of the mirror slope trace measured with the LTP from the desired slope distribution.

The functions $f_{1}(x)$ and $f_{2}(x)$ are a priori unknown functions, which we determine by approximation based on a set of preliminary LTP measurements with the mirror. This approach is similar to one used in Ref. 5 for the tuning of KB mirrors already installed in a beamline. Below, we provide the mathematical scope of the approach.

Consider the slope of an ideal elliptical surface in the same notation:

$$
\alpha^{0}(x, \hat{C})=C_{0}^{0}+C_{1}^{0} f_{1}(x)+C_{2}^{0} f_{2}(x)
$$


Deviations from the ideal surface slope may be expressed:

$$
\Delta \alpha(x, \hat{C})=\Delta C_{0}+\Delta C_{1} f_{1}(x)+\Delta C_{2} f_{2}(x)
$$

where $\Delta C_{0}=C_{0}-C_{0}^{0}, \Delta C_{1}=C_{1}-C_{1}^{0}$, and $\Delta C_{2}=C_{2}-C_{2}^{0}$.

The LTP measurements of the optic provide us with slope traces over a discrete set of positions $\left\{x_{i}\right\}$ in the tangential direction. Each slope point $i$ is measured with a final error $\varepsilon_{i}$. Therefore, the result of a slope trace measurement for a given set of adjustments of the mirror $C_{i}$ can be expressed with a trace of slope deviations from an ideal (desired) shape:

$$
\delta \alpha_{0}\left(x_{i}\right)=\Delta C_{0}+\Delta C_{1} f_{1}\left(x_{i}\right)+\Delta C_{2} f_{2}\left(x_{i}\right)+\varepsilon_{0 i}
$$

In Eq. (10) we use the lower index that is 0 for slope deviation $\delta \alpha_{0}\left(x_{i}\right)$ and for the error term $\varepsilon_{0 i}$ to denote an index number of a measurement. The next measurement (with the index number 1) is assumed to be performed when one of the mirror adjustments was changed, say the left bending moment, represented by $C_{1}$, by $\delta C_{1}$ :

$$
\delta \alpha_{1}\left(x_{i}\right)=\Delta C_{0}+\left(\Delta C_{1}+\delta C_{1}\right) f_{1}\left(x_{i}\right)+\Delta C_{2} f_{2}\left(x_{i}\right)+\varepsilon_{1 i}
$$

We may subtract these two measurements, Eq. (10) from Eq (11), and neglecting measurement error $\left(\varepsilon_{1 i}-\varepsilon_{0 i}\right)$, we may solve for an approximation $f_{1}^{*}\left(x_{i}\right)$ to $f_{1}\left(x_{i}\right)$,

$$
f_{1}^{*}\left(x_{i}\right) \approx\left[\delta \alpha_{1}\left(x_{i}\right)-\delta \alpha_{0}\left(x_{i}\right)\right] / \delta C_{1} .
$$

With the asterisk we separate the estimate from the true value of the function. We repeat the approximation procedure for $f_{2}\left(x_{i}\right)$ by taking one measurement (with index number 2 ) when 
the other mirror adjustment was changed, in this case the right bending moment, represented by $C_{2}$, by $\delta C_{2}$ :

$$
f_{2}^{*}\left(x_{i}\right) \approx\left[\delta \alpha_{2}\left(x_{i}\right)-\delta \alpha_{0}\left(x_{i}\right)\right] / \delta C_{2} .
$$

Functions $f_{1}^{*}\left(x_{i}\right)$ and $f_{2}^{*}\left(x_{i}\right)$ are approximations of the bender characteristic functions experimentally measured over the set of discrete positions. Using these functions $f_{1}^{*}\left(x_{i}\right)$ and $f_{2}^{*}\left(x_{i}\right)$, linear regression analysis can be applied to the set of equations

$$
\delta \alpha_{0}\left(x_{i}\right)=\Delta C_{0}+\Delta C_{1} f_{1}^{*}\left(x_{i}\right)+\Delta C_{2} f_{2}^{*}\left(x_{i}\right)
$$

to find the best approximation to the optimal adjustment parameters that correspond to the minimum of the mean square variation of $\delta \alpha_{0}\left(x_{i}\right) \cdot{ }^{10-14}$ First, the regression matrix may be formulated:

$$
\hat{A} \approx\left[\begin{array}{ccc}
1 & f_{1}^{*}\left(x_{1}\right) & f_{2}^{*}\left(x_{1}\right) \\
1 & f_{1}^{*}\left(x_{2}\right) & f_{2}^{*}\left(x_{2}\right) \\
\vdots & \vdots & \vdots \\
1 & f_{1}^{*}\left(x_{m}\right) & f_{2}^{*}\left(x_{m}\right)
\end{array}\right],
$$

where $m$ is total number of points measured in a slope trace.

This provides a solution ${ }^{10-14}$ for the best approximation to the optimal adjustment parameters:

$$
\Delta \hat{C}^{*}=\left(\hat{A}^{\prime} \hat{A}\right)^{-1} \hat{A}^{\prime} \delta \alpha_{0}\left(x_{i}\right),
$$

with an estimation for the dispersion of these parameters:

$$
D\left(\Delta \hat{C}^{*}\right)=\sigma^{2}\left(\hat{A}^{\prime} \hat{A}\right)^{-1}
$$


The dispersion parameter $\sigma^{2}$ can be estimated from:

$$
\sigma^{2} \approx(m-p)^{-1} \sum_{i}\left[\delta \alpha_{0}\left(x_{i}\right)-\Delta C_{0}^{*}-\Delta C_{1}^{*} f_{1}^{*}\left(x_{i}\right)-\Delta C_{2}^{*} f_{2}^{*}\left(x_{i}\right)\right]^{2}
$$

Where $p$ is the number of parameters plus 1 . In our case $p=4$.

Without the offset term $C_{0}$, our presentation above is exactly that which has been very briefly outlined in the literature. ${ }^{5}$ Note, that without this term, one cannot get the right parameters for bender settings while using the $\operatorname{method}^{5}$ for tuning optics at a beamline. Although this term is important to the accuracy of the application of the method, the most important difference from previous work is that the method is extended to be applicable to the in-lab tuning based on slope data generated by the LTP before the optic is placed in the beamline. ${ }^{15}$

\section{Reduction to an empirical and intuitive tuning method}

Previously in our lab, in order to find the optimal settings of bending couples of a mirror, we compared (using the LTP-II software) a height distribution obtained by the integration of slope data measured with the LTP with an ideal shape desired for the mirror. A difference trace obtained by subtracting the ideal (desired) trace from the measured one and a root mean square variation of the difference trace were used as criteria for a decision about quality of tuning and value of change of the settings to be made by an operator. Because two settings should be found, this was a procedure that was mostly based on intuition and experience of the operator. Sometimes, there were 50-60 changes of the bender settings with followed slope measurements before we got an acceptable mirror shape. At first glance, it seems to be possible to simply improve the tuning procedure by incorporating directly a least square fitting of the measured trace data to an ellipse after each adjustment of the bender at the LTP. With the known 
parameters (there are three instead of one parameter of the rms variation of the difference trace) of the best fit ellipse, operator would have a better clue for faster tuning of a mirror. However, the minimal total number of parameters of the fit is 5 not 3 , including overall tilt of the measured mirror surface and position of the effective center of the mirror elliptical segment. As a result, in a general case of the LTP measurement with a precision of $\sim 0.5 \mu \mathrm{rad}$ (rms), achieving a reliable fit for grazing incidence geometry is difficult even with a method which is designed to give a unique elliptical fit. ${ }^{16}$ Moreover, the fitting methods, to best our knowledge, do not provide the standard errors of the best fit ellipse parameters ${ }^{17}$ and, therefore, it is impossible to clarify the appropriateness of the fit.

Below we consider a reduction of the technique described in Sec. 2 to a more empirical tuning method that allows to an operator to less intuitively and more rapidly tune and characterize bendable optics. In some sense, the reduced method is essentially an average over the detailed procedure described above. Indeed, in order to find only three parameters $C_{0}, C_{1}$ and $C_{2}$ our analysis in Sec. 2 uses an over-determined set of equations (14) by considering each point in the slope data. Of course, use of the over-determined set of equations has a strong sense, because with regression analysis we significantly decrease errors for the found parameters by effective averaging over whole number of the trace points, each measured with a relatively low accuracy. Another approach that could provide a comparable accuracy would be based on a lesser number of equations that are built for significantly averaged measurables. As the averaged measurables in the reduced method, we use a smaller number of surface curvatures evaluated on large subsets of the surface slope data. 
Recalling equation (4) we see that surface curvature is a linear function of two setting parameters, $C_{1}$ and $C_{2}$. Therefore, a value of curvature averaged over a tangential segment of the mirror length also satisfies a linear relation, similar to (4):

$$
\langle C u r\rangle_{A} \equiv\left\langle\frac{d^{2} y}{d x^{2}}\right\rangle_{A}=C_{1}\left\langle g_{1}\left(x_{i}\right)\right\rangle_{A}+C_{2}\left\langle g_{2}\left(x_{i}\right)\right\rangle_{A},
$$

where averaging denoted with the angle brackets is performed over a segment $A$ of the total mirror length. The values of the averaged functions $g_{1, A} \equiv\left\langle g_{1}\left(x_{i}\right)\right\rangle_{A}$ and $g_{2, A} \equiv\left\langle g_{2}\left(x_{i}\right)\right\rangle_{A}$ are the constants characteristic for the segment $A$ of a particular bendable mirror, and do not depend on the values of the bender settings, $C_{1}$ and $C_{2}$.

Similar to the characteristic functions $f_{1}(x)$ and $f_{2}(x)$ used in the generalized technique described in Sec. 2, the values of the constants $g_{1, A}$ and $g_{2, A}$ that are a priori unknown can be determined based on a sequence of three measurements with sequential change of the bender settings by $\delta C_{1}$ and $\delta C_{2}$ :

$$
\begin{aligned}
& \langle\text { Cur }\rangle_{0, A}=C_{1} g_{1, A}+C_{2} g_{2, A}, \\
& \langle C u r\rangle_{1, A}=\left(C_{1}+\delta C_{1}\right) g_{1, A}+C_{2} g_{2, A}, \\
& \langle C u r\rangle_{2, A}=C_{1} g_{1, A}+\left(C_{2}+\delta C_{2}\right) g_{2, A} .
\end{aligned}
$$

Then,

$$
g_{1, A}=\left(\langle C u r\rangle_{1, A}-\langle C u r\rangle_{0, A}\right) / \delta C_{1} \text { and }
$$




$$
g_{2, A}=\left(\langle\text { Cur }\rangle_{2, A}-\langle\text { Cur }\rangle_{0, A}\right) / \delta C_{2} .
$$

If the constants in Eq. (19) are known, in order to uniquely find two bender settings $C_{1}^{0}$ and $C_{2}^{0}$ corresponding to the desired mirror shape, one need only two equations, like Eq. (19), for two uncrossed (uncorrelated) segments $A$ and $B$ of the mirror:

$$
\begin{aligned}
& \langle\operatorname{Cur}\rangle_{A}^{0}=C_{1}^{0}\left\langle g_{1}\left(x_{i}\right)\right\rangle_{A}+C_{2}^{0}\left\langle g_{2}\left(x_{i}\right)\right\rangle_{A}, \\
& \langle C u r\rangle_{B}^{0}=C_{1}^{0}\left\langle g_{1}\left(x_{i}\right)\right\rangle_{B}+C_{2}^{0}\left\langle g_{2}\left(x_{i}\right)\right\rangle_{B} .
\end{aligned}
$$

In Eqs. (22), $\langle C u r\rangle_{A}^{0}$ and $\langle C u r\rangle_{B}^{0}$ are the averaged curvatures evaluated for the same sets of points of the segments $A$ and $B$ of the ideal (desired) surface. In order to find the constants $g_{1, B}$ and $g_{2, B}$, the same set of three LTP slope measurements over the entire surface can be used.

Practically, for each measured slope trace (as well for the ideal trace), we calculate four curvatures $\left(\right.$ radii $\left.^{-1}\right)$ for three different segments of the surface and for the entire clear aperture of the mirror - Fig. 3. In order to minimize errors the measured mirror slopes are fitted to a 5th order polynomial with removal of piston and tilt. Numerical experiments have shown that this is the proper balance between accuracy in the succeeding analysis, and adding non-existent features to the surface that can be due to the noise in the LTP. This polynomial is next integrated into the height of the surface, to 6th order. Proceeding this way, rather than a straightforward integration of the discrete slope traces gives a less error prone estimation of the surface height. Next, the set of heights is divided into 3 roughly equal segments. We fit, using a matrix-based least squares method analogous to that described above, a radius to the entire surface, and to each of the three segments. By standard propagation of error methods a standard error is assigned to each radius. 
The magnitudes of the parameters $g_{1, j}$ and $g_{2, j}$, where indexes $j$ denote the left-hand, $j \equiv L$, the central, $j \equiv C$, the right-hand, $j \equiv R$, one-third parts of the clear aperture, and the total clear aperture of the mirror, $j \equiv T$, can be found experimentally from a few measurements at different settings $C_{1}$ and $C_{2}$ (Figs. 4 and 5) as the slopes of the corresponding linear dependences. Then the optimal settings are found by directly solving a set of two equations (22) corresponding to two different surface segments, say, left-hand and the right hand segments in Fig. 3. For a cross check and comparison to the ideal shape we fit the exact same four regions using the ideal ellipse based on the desired parameters of $r, r^{\prime}$ and $\theta$ - see Fig. 1 . In order to treat the ideal shape just like the data, we use the exact derivative of the ellipse equation, fit a 5 th order polynomial to it, and integrate the slope to 6th order. Applying this approximation to the ideal slope in exactly the same manner as to the measured trace provides the same errors and therefore does not perturb the adjustment. At each step the operator can double check whether the left or right bender mechanism should be tightened or loosened to achieve the proper figure. The center radius and the total radius allow the adjuster to keep the overall radius in perspective while adjusting the left and right parts of the bender mechanism.

\section{Application to tune a KB mirror}

In the metrology lab we find for properly designed bendable mirrors ${ }^{7}$ the two adjustments are to $0^{\text {th }}$ order independent of one another, see also Ref. 7. Thus the left adjustment mainly affects the left curvature, and the right adjustment the right curvature. Therefore, extrapolation of the calibration graphs (Figs. 4, 5) provides a good approximation of the optimal settings. Such graphs are typically provided with the metrology report showing the dependence of all four of the radii on the encoder readouts from the left and right adjustments. If there are any unintended 
mechanical constraints from design or assembly they typically show up at this point as inconsistencies in the calibration data. Backlash or changes over the time seen in the metrology lab allow repairs to be made before the mirror is moved to the beamline. Typically, the adjustments are made in both forward and reverse directions to highlight any hysteresis effects. Trials in the metrology lab have shown this to be a rapid, productive means of adjusting elliptical bendable substrates. We estimate the new procedure provides a reduction in effort of a factor of approximately 10 with respect to the older method of sequentially fitting ellipses. Significantly, we have readjusted mirrors to another desired elliptical shape in the beamline without dis-assembly, in order to change the focal imaging distance based on previous measurements at the metrology lab.

As an illustration of the application of the developed technique, we take the case of a bendable mirror from Beamline 8.0.1.1 at the ALS. At one geometry of use it is designed to have conjugate distances of $r=26.8 \mathrm{~m}$ and $r^{\prime}=3.0 \mathrm{~m}$. The grazing angle of incidence is $\theta=0.05236 \mathrm{rad}$ ( 3 degrees). The four best fitted radii of the segments as they are shown in Fig. 3 and corresponding to the desired tangential shape of the mirror surface are presented in Table 1. We start tuning process with the mirror adjusted to a slightly pre-bended shape that was measured with the LTP in the 1st scan - Table 1. In the course of the 1st scan, the settings of the upstream and downstream bender encoders were 105.825 counts, and 126.294 counts, respectively. Note that for the application of the described tuning technique, it is does not matter what are the absolute values or units of the coupling moment applied to the sides of the mirror substrate. The only requirement for the encoder is that it provide a linear response to the coupling. 
In order to get a prediction for the mirror bender settings to be applied in the first step of the tuning process, we performed two additional scans with a sequential change of each setting. The magnitudes of the corresponding settings as they are monitored with the upstream, $C_{U S}$, and downstream bender encoders, $C_{D S}$, are presented in Table 2 together with new encoder settings calculated using the algorithm discussed in Sec. 2. An LTP measurement (that is the $4^{\text {th }}$ scan) with the mirror adjusted to the calculated settings completes the first tuning cycle. After the $1^{s t}$ tuning, the radii of the mirror segments were measured to be significantly closer to the desired values than it was originally - Table 1 ; however, there is a noticeable difference that is larger than the fitting errors listed in Table 2. The most probable reason for the difference is a nonlinearity of the mirror bender mechanism that has rather complicated mechanical design with a number of joints and strongly stressed elements.

In order to get a mirror surface shape closer to the desired ellipse, we repeat the tuning cycle. In the second tuning in order to find new approximations for the mirror characteristic functions (12) and (13), we use the $4^{\text {th }}$ scan (that was already performed) and two additional scans, the $5^{\text {th }}$ and $6^{\text {th }}$ scans, with bender settings appropriately changed with respect to the settings for the $4^{\text {th }}$ scan - Table 2. A control measurement (the $7^{\text {th }}$ scan in Table 2) was performed with the mirror adjusted to the calculated settings completes after the second tuning cycle. The radii best fitted to the resulting surface are very close to the desired values - Table 2 . The tiny difference that is also larger than the fitting errors is probably due to a small error of sagittal shaping of the mirror substrate that makes it impossible in principle to exactly tune the mirror. 
As a check for fast convergence of the tuning procedure with the particular mirror under investigation, we did a third iteration which gave values $\delta C_{U S}=1.04$ counts and $\delta C_{D S}=-1.15$ counts for the third tuning of the settings $C_{U S}$ and $C_{D S}$. These numbers are only about $5 \%$ of that of the second tuning and about $1 \%$ of setting changes made in the course of the $1^{\text {st }}$ tuning cycle. The best fitted radii corresponding to the $3^{\text {rd }}$ tuning (Table 1) confirm that we have reached the limit to the adjustment with this bendable mirror and the current accuracy of the LTP measurements.

Ray traces based on the slope data also show no essential difference when these last small adjustments are implemented. Figure 6 depicts a wavefront ray trace based on the last adjustments above. Calculations follow the equations for ray deviations based on aberration coefficients used by many authors. ${ }^{15,18}$ Documentation of this code is in progress. We have moved the $r^{\prime}$ distance to $2896 \mathrm{~mm}$ instead of $3000 \mathrm{~mm}$ in order to zero out the defocus term, and show the best possible performance, as would be adjusted on the beamline by varying $r^{\prime}$ a small amount. Since the final parameters of use in the beamline are typically not this well controlled, final in situ adjustments would be made at the beamline. We feel going to a slightly different $r^{\prime}$ for the ray trace shows more exactly the performance that would be achieved in practice. Clearly the symmetry of the pattern shows that the coma term is small, only $10 \%$ of the remaining aberration. $90 \%$ of the aberration is 4 th order and higher. These aberrations are not removable with a two couple uniform bendable mirror without adding a variation in the cross section of the mirror. 


\section{Summary}

We have reviewed the basic theory of tangential elliptical benders and shown two in-lab LTP-based methods of adjustment of bending couples, both of which are easier, and faster than previous methods. These methods are based on the basic linearity of the bending process. We also have developed a simple way of monitoring the bending at each step in the process by ray trace calculation. Both of these methods, and the monitoring scheme use only the geometry of use, and the slope data from the Long Trace Profiler. The methods provide a complete calibration of the bendable mirror which can be used after installation to reset the mirror to different conjugate distances. The next step in the systematic improvement of optical performance for the application of KB pairs in synchrotron beamlines will be to more tightly control $r, r^{\prime}$ and $\theta$ for mirror installation so that the metrology/adjustment process may be completely integrated. We believe a completely integrated approach, where the metrology and beamline alignment are not arbitrarily separated, is the proper approach to systematic improvement of beamline performance. ${ }^{19-21}$ 


\section{Acknowledgements}

The authors are grateful to Amparo Rommeveaux and Howard Padmore for useful discussions. The Advanced Light Source is supported by the Director, Office of Science, Office of Basic Energy Sciences, Material Science Division, of the U.S. Department of Energy under Contract No. DE-AC02-05CH11231 at Lawrence Berkeley National Laboratory.

\section{Disclaimer}

This document was prepared as an account of work sponsored by the United States Government. While this document is believed to contain correct information, neither the United States Government nor any agency thereof, nor The Regents of the University of California, nor any of their employees, makes any warranty, express or implied, or assumes any legal responsibility for the accuracy, completeness, or usefulness of any information, apparatus, product, or process disclosed, or represents that its use would not infringe privately owned rights. Reference herein to any specific commercial product, process, or service by its trade name, trademark, manufacturer, or otherwise, does not necessarily constitute or imply its endorsement, recommendation, or favoring by the United States Government or any agency thereof, or The Regents of the University of California. The views and opinions of authors expressed herein do not necessarily state or reflect those of the United States Government or any agency thereof or The Regents of the University of California. 


\section{$\underline{\text { Biographies }}$}

Wayne R. McKinney received his $\mathrm{BA}, \mathrm{MA}$, and $\mathrm{PhD}$ in Physics from The Johns Hopkins University, finishing in 1974 with a thesis in ultraviolet astronomy. He then completed a post-doctoral appointment in molecular biology supported by a National Cancer Institute Fellowship at the Biology Department of Brookhaven National Laboratory in 1977. Remaining at Brookhaven from 1977 to 1979 in the Instrumentation Division he designed optical systems for the National Synchrotron Light Source. From 1979 to 1987 he joined the research staff of the Richardson Grating Lab in Rochester New York, becoming Manager of Diffraction Grating R\&D in 1981. From 1987 to 1989 he was a staff scientist in the Center for X-Ray Optics at Lawrence Berkeley National Laboratory working on water cooled optical components and monochromator designs for the Advanced Light Source. This work won local and national Tech Transfer Awards, and led to Fellow status in the OSA. He now works directly for the Advanced Light Source where he designed and built the first infrared beamlines. Currently his responsibilities are in optical metrology, particularly the specification of $x$-ray optics by calculation of scattering.

Jonathan Kirschman received his BA in Electrical Engineering and Computer Science with a minor in Bioengineering from Univeristy of California at Berkeley in 2006. He then worked as Baccalaureate fellow and then as a Research Associate at the Optical Metrology Lab at Lawrence Berkeley National Laboratory. There he worked on improving optical metrology instrumentation and characterizing state of the art X-ray optics. He is currently teaching overseas in Japan. His research interests are in biomedical engineering and optics. 
Alastair A. MacDowell received his $\mathrm{BSc}$ and $\mathrm{PhD}$ in physical chemistry from the University of Manchester (UK) in 1976 and 1980 respectively. He has worked in the development and application of various instrumental techniques used at the several synchrotron facilities. He has worked at: Daresbury (UK), Brookhaven (USA) and now Berkeley (USA). His current interests are the deployment of a new aberration corrected photoelectron emission microscope and the development of a facility for hard x-ray micro-tomography .

Tony Warwick graduated in the UK in 1979 and has since worked in various programs at Lawrence Berkeley National Laboratory. In Nuclear Science, Accelerator Physics, Fusion Research and at the Advanced Light Source, where he is currently deputy for the Experimental Systems Group. His research interests include x-ray microscopy and the associated x-ray beamlines and insrtumentation.

Valeriy V. Yashchuk received his MS degree in experimental physics from St. Petersburg State University (Russia) in 1979, and his PhD degree from St. Petersburg Nuclear Physics Institute (Russia) in 1994. He is currently leading the Optical Metrology Laboratory at the Advanced Light Source, Lawrence Berkeley National Laboratory. He has authored more than 50 scientific articles in the fields of atomic and molecular physics, nonlinear optics, electro- and magneto-optics, laser spectroscopy, experimental scientific methods and instrumentation, and optical metrology. In 1986 for the development of a method of reduction of phase space of an atomic beam he was awarded the Leningrad Komsomol Prize in physics. In 2007, he received R\&D Magazine's R\&D 100 Award for development of Laser-Detected MRI. His current research interest is in x-ray optics, optical instrumentation and metrology for x-ray optics. 


\section{Figure captions:}

Figure 1: The geometry of a tangential elliptical mirror.

Figure 2: $C_{1}, C_{2}$ represent the applied couples. $C_{2}$ is typically the downstream adjustment. Arrows define positive couples.

Figure 3: Typical locations of the four radii for fitting. The clear aperture is only measured and shown. The total radius uses all the points. Each of the other 3 radii uses only $\sim 1 / 3$ of the points.

Figure 4: Plot of curvature with respect to the upstream (left) encoder voltage for the upstream bending couple for fixed downstream (right) setting, for a typical mirror measurement. The left (upstream) curvature shows significant variation, and the right (downstream) curvature shows much less, as expected. The observed linearity of curvature with bending validates the model.

Figure 5: Plot of curvature with respect to encoder voltage for the downstream bending couple for a typical mirror measurement. Right (downstream) curvature shows significant variation, and left (up) curvature shows much less, as expected. The observed linearity of curvature with bending validates the model.

Figure 6: Wavefront ray trace simulation of beamline performance of the final adjusted mirror for the ALS beamline 8.0.1.1. A perfect point source was assumed.

\section{Table captions}

Table 1: Radii of the mirror surface segments after $1^{\text {st }}, 2^{\text {nd }}$, and $3^{\text {rd }}$ tuning cycles. 
Table 2: Settings of the mirror benders in the $1^{\text {st }}, 2^{\text {nd }}$, and $3^{\text {rd }}$ tuning cycles. and in row 10 the settings after the $3^{\text {rd }}$ tuning. They do not differ significantly from the settings at the end of the $2^{\text {nd }}$ tuning in row 7.

\section{Figure 1:}

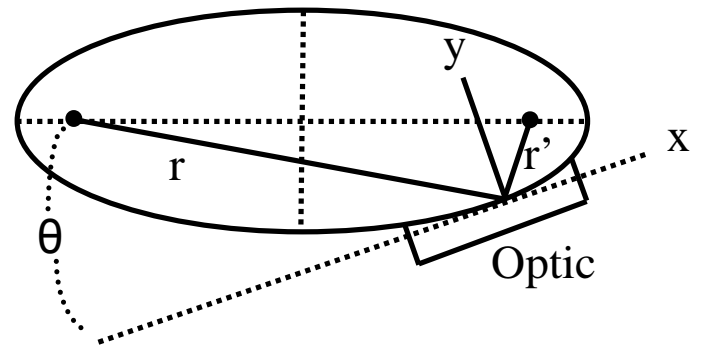

Figure 2:

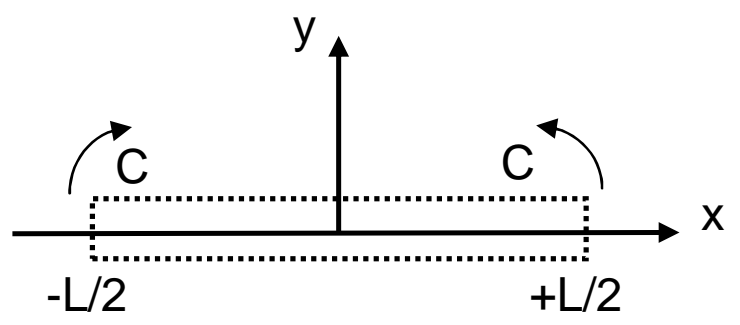


Figure 3:

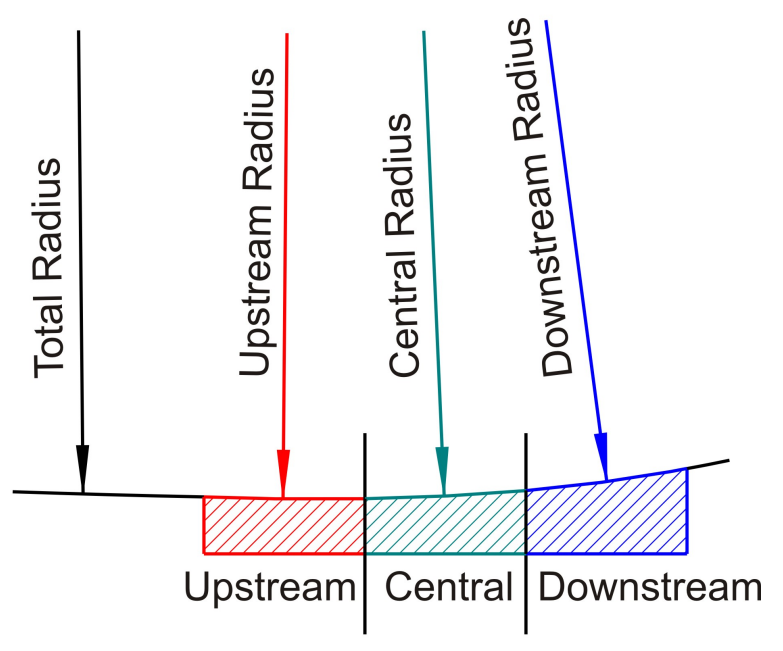


Figure 4:

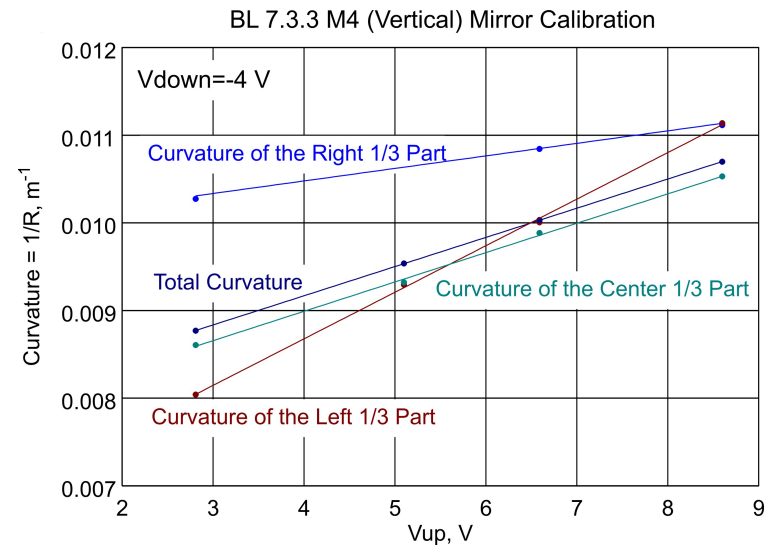

Figure 5:

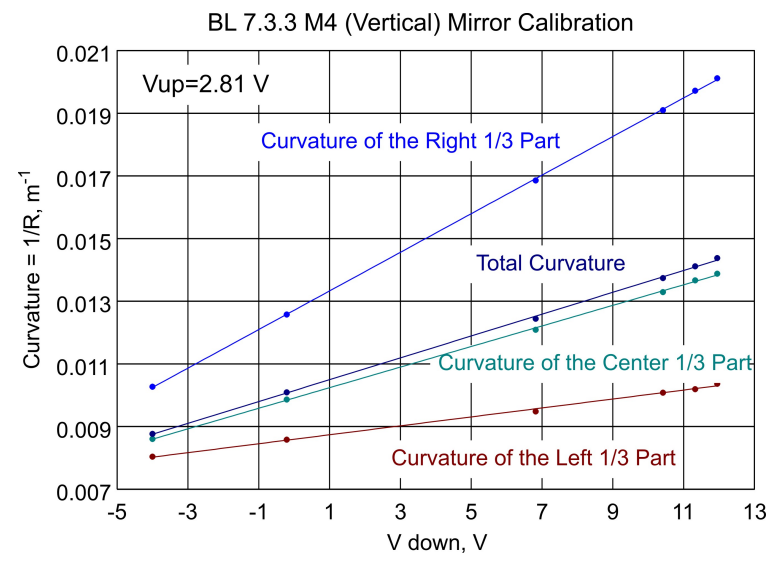


Figure 6:

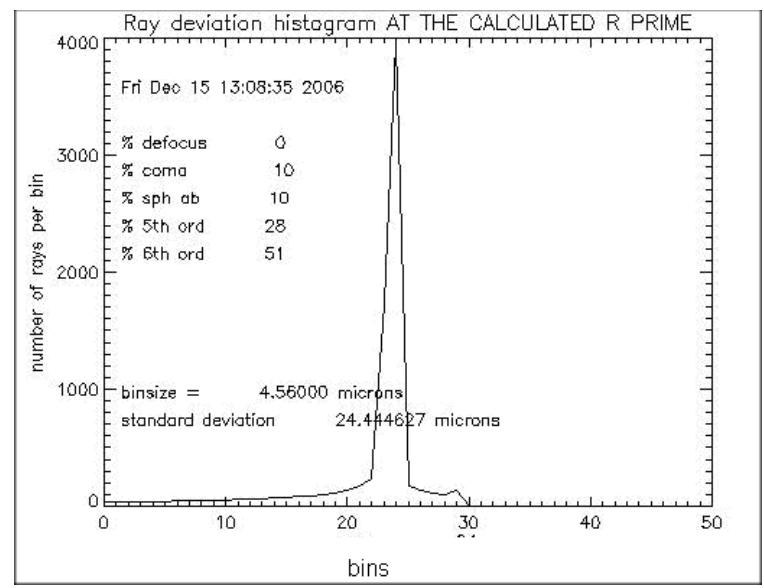

Table 1:

\begin{tabular}{|l|l|c|r|c|c|}
\hline & Desired shape & \multicolumn{1}{|c|}{$1^{\text {st }}$ scan } & \multicolumn{1}{c|}{$1^{\text {st }}$ tuning } & $2^{\text {nd }}$ tuning & $3^{\text {rd }}$ tuning \\
\hline $\mathrm{R}_{\mathrm{T}}[\mathrm{m}]$ & $102.8 \pm 0.05$ & $211.0 \pm 0.10$ & $104.6 \pm 0.18$ & $102.49 \pm 0.06$ & $102.44 \pm 0.06$ \\
\hline $\mathrm{R}_{\mathrm{L}}[\mathrm{m}]$ & $105.7 \pm 0.03$ & $220.9 \pm 0.26$ & $117.0 \pm 0.19$ & $107.00 \pm 0.08$ & $106.86 \pm 0.08$ \\
\hline $\mathrm{R}_{\mathrm{C}}[\mathrm{m}]$ & $102.9 \pm 0.03$ & $209.6 \pm 0.05$ & $104.6 \pm 0.11$ & $102.28 \pm 0.04$ & $102.23 \pm 0.04$ \\
\hline $\mathrm{R}_{\mathrm{R}}[\mathrm{m}]$ & $100.0 \pm 0.03$ & $210.6 \pm 0.11$ & $96.6 \pm 0.06$ & $100.12 \pm 0.01$ & $100.30 \pm 0.01$ \\
\hline
\end{tabular}


Table 2:

\begin{tabular}{|c|c|c|c|}
\hline scan & $C_{U P}$ [counts] & $C_{D S}$ [counts] & \multirow{2}{*}{$1^{\text {st }}$ tuning } \\
\hline 1 & 105.83 & 126.29 & \\
\hline 2 & 115.83 & 126.29 & \\
\hline 3 & 105.83 & 136.29 & \\
\hline 4 & 189.27 & 278.42 & \multirow{2}{*}{$2^{\text {nd }}$ tuning } \\
\hline 5 & 199.27 & 278.42 & \\
\cline { 1 - 3 } 6 & 189.27 & 288.42 & \multirow{2}{*}{$3^{\text {rd }}$ tuning } \\
\hline 7 & 217.66 & 257.86 & \\
\hline 8 & 227.66 & 257.86 & \\
\hline 9 & 217.66 & 267.86 & \multirow{2}{*}{ After $3^{\text {rd }}$ tuning } \\
\hline 10 & 218.70 & 256.71 & \multicolumn{2}{|c}{} \\
\hline
\end{tabular}

1. W. Yun, M. Feser, A. F. Lyon, F. Duewer and Y. Wang, Pathways to sub-10nm x-ray imaging using zone plate lens, SPIE Proceedings 5539, pp. 133-137 (2004).

2. V. Nazmov, E. Reznikova, M. Boerner, J. Mohr, V. Saile, A. Snigerev, I. Snigireva, M. Dimichiel, M. Drakopoulos, R. Simon and M. Grigoriev, Refractive lenses fabricated by deep SR lithography and LIGA technology for X-ray energies from $1 \mathrm{keV}$ to $1 \mathrm{MeV}$, AIP Proceedings 705, pp. 752-755 (2004).

3. T. Warwick, N. Andresen, J. Comins, A. Franck, M. Gilles, T. Tonnessen and T. Tyliszczak, Large aperture micro-focus KB mirrors for spectroscopy experiments at the Advanced Light Source, AIP Proceedings 705, pp. 772-775 (2004).

4. P. Kirkpatrick and A. V. Baez, "Formation of optical images by X-rays," J. Opt. Soc. Am. 38(9), 766-774 (1948).

5. O. Hignette, A. Freund and E. Chinchio, Incoherent X-ray mirror surface metrology, SPIE Proceedings 3152, pp. 188-199 (1997). 
6. M. R. Howells and D. Lunt, "Design considerations for adjustable-curvature, high-power, X-ray mirrors based on elastic bending," Optical Engineering 32(8), 1981-1989 (1993).

7. M. R. Howells, D. Cambie, R. M. Duarte, S. Irick, A. Mac Dowell, H. A. Padmore, T. Renner, R. Seungyu and R. Sandler, "Theory and practice of elliptically bent X-ray mirrors," Optical Engineering 39(10), 2748-2762 (2000).

8. R. J. Roark, Formulas for Stress and Strain, McGraw-Hill, New York (1964).

9. S. Ferrer, M. Krisch, F. De Bergevin and F. Zontone, "Evaluation of the anticlastic curvature of elastically bent crystals for X-ray focusing optics," Nuclear Instruments and Methods in Physics Research Section A: Accelerators, Spectrometers, Detectors and Associated Equipment 311(3), 444-447 (1992).

10. V. V. Yashchuk, Positioning errors of pencil-beam interferometers for long trace profilers, SPIE Proceedings 6317, pp. 63170A-63112 (2006).

11. R. L. Plackett, Principles of Regression Analysis, Clarendon Press, Oxford (1960).

12. D. Hudson, Lectures on Elementary Statistics and Probability, CERN, Geneva (1963).

13. J. Neter and W. Wasserman, Applied Linear Statictical Models, Inwin-Dorsey International, London (1974).

14. M. Kendall and A. Stuart, The Advanced Theory of Statistics, Oxford University Press, New York (1979).

15. W. R. Mc Kinney, S. C. Irick, J. L. Kirschman, A. A. Mac Dowell, T. Warwick and V. V. Yashchuk, New procedures for the adjustment of elliptically bent mirrors with the long trace profiler, SPIE Proceedings 6704, p. 67040G (2007). 
16. A. Fitzgibbon, M. Pilu and R. B. Fisher, "Direct least square fitting of ellipses," IEEE Transactions on Pattern Analysis and Machine Intelligence 21(5), 476-480 (1999).

17. G. A. Watson, "Least squares fitting of circles and ellipses to measured data," Bit 39(1), 176-191 (1999).

18. H. A. Padmore, M. R. Howells and W. R. Mc Kinney, "Grazing-Incidence Monochromators for Third Generation Synchrotron Radiation Sources," in Vacuum Ultraviolet Spectroscopy II, Samson and Ederer, Eds., Academic Press (1998).

19. V. V. Yashchuk, E. L. Church, M. Howells, W. R. Mc Kinney and P. Z. Takacs, "21st Century Metrology for Synchrotron Radiation Optics - Understanding How to Specify and Characterize Optics," in The 3rd International Workshop on Metrology for X-ray Optics, Pohang Accelerator Laboratory, Daegu, Korea (2006).

20. E. L. Church and P. Z. Takacs, "Specification of surface figure and finish in terms of system performance," Applied Optics 32(19), 3344-3353 (1993).

21. E. L. Church and P. Z. Takacs, "Specification of glancing- and normal-incidence X-ray mirrors," Optical Engineering 34(2), 353-360 (1995). 